\title{
Tamizaje fitoquímico de extractos obtenidos de la planta Sapindus saponaria L que crece en Cuba
}

\author{
Phytochemical screening of extracts obtained from the Sapindus saponaria L plant that grows \\ in Cuba
}

\section{Anairis Pujol Garcia ${ }^{1 a^{*}}$, Beatriz Tamargo ${ }^{1 b}$, Eva Salas $^{1}$, Camilo Calzadilla $^{1}$, Reinaldo Acevedo ${ }^{1}$, Gustavo Sierra $^{2}$}

\begin{abstract}
Resumen: Las plantas medicinales son consideradas una fuente importante de componentes biológicamente activos, que pueden ser usados en el tratamiento de disímiles enfermedades. En particular el potencial medicinal del género Sapindus $L$. ha sido estudiado por autores de varios países y dentro de este género, la especie Sapindus saponaria $L$ se ha aplicado en el tratamiento de diversas enfermedades. Para la especie S. saponaria $L$ se refieren 32 usos y 14 efectos biológicos comprobados. Dentro de los efectos biológicos más referenciados están acaricida, antihemorrágico, anti protozoario, antiulceroso, citotóxico, molusquicida y nematicida. Además, el pericarpio del fruto es la parte más empleada, aunque también se han empleado las hojas, ramas, corteza y semillas. Los principales efectos biológicos se encuentran relacionados con elevados contenidos de saponinas y flavonoides en los extractos obtenidos de diferentes partes de la planta. El objetivo de este trabajo es determinar los principales componentes de la planta Sapindus saponaria $L$ que crece en Cuba mediante el tamizaje fitoquímico de las diferentes partes de la planta. Para ello, se realizó una marcha experimental con extracciones alcohólicas y acuosas del pericarpio del fruto, la corteza del tallo y hojas de la planta. Los resultados del tamizaje en ambas extracciones y análisis de componentes revelaron que el pericarpio del fruto y hojas son ricas en flavonoides mientras que el pericarpio del fruto posee un alto contenido de saponinas. La composición de Sapindus saponaria L que crece en Cuba es similar a la composición de S. saponarias que crecen en otras regiones de Latinoamérica.
\end{abstract}

Palabras clave: Sapindus saponaria L, saponinas, flavonoides, tamizaje fitoquímico.

Abstract: Medicinal plants are considered an essential source of biologically active components, which can be used in the treatment of different diseases. In particular, the medicinal potential of the genus Sapindus $L$. has been studied by authors from various countries, and within this genus, the species Sapindus saponaria $L$ has been applied in the treatment of various diseases. For the S. saponaria $\mathrm{L}$ species, 32 uses and 14 proven biological effects refer. Among the most referenced biological effects are acaricide, antihemorrhagic, anti-protozoan, antiulcer, cytotoxic, molluscicide, and nematicide. Besides, the pericarp of the fruit is the most used part, although the leaves, branches, bark, and seeds have also been used. The main biological effects are related to the high content of saponins and flavonoids in the extracts obtained from different parts of the plant. The objective of this work is to determine the main components of the Sapindus saponaria $L$ plant that grows in Cuba, employing phytochemical screening of the different parts of the plant. For this, an experimental run was carried out with alcoholic and aqueous extractions of the fruit pericarp, the stem bark, and leaves of the plant. The results of the screening in both extractions and component analysis revealed that the rind of the fruit and leaves are rich in flavonoids while the pericarp of the fruit has a high content of saponins. The composition of Sapindus saponaria $L$ that grows in Cuba is similar to the composition of $S$. Saponaria that grow in other regions of Latin America.

Key words: Sapindus saponaria L, saponins, flavonoids, phytochemical screening.

\section{Introducción}

Sapindus saponaria $\mathrm{L}$. es un árbol que puede alcanzar tamaño de 18-20 metros de altura, aunque comúnmente en zonas urbanas alcanza una altura de aproximadamente 12 metros. Posee un tronco más o menos cilíndrico con diámetros de $40-45 \mathrm{~cm}$, de corteza color gris claro, lenticelada cuando joven, un poco lisa, con pequeñas oquedades, tornándose finamente agrietada y escamosa en el estado adulto. Posee una copa globosa e irregular con amplitud de 8-10 m. La Sapindus saponaria L. originaria de América tropical, se distribuye desde el sur de Estados Unidos, Centroamérica, Venezuela, Ecuador, Perú, Colombia y Brasil. Ha sido introducida además en los trópicos del viejo mundo, a las islas Filipinas, parte de Oceanía y el oeste de la India. En Colombia se encuentra en los cañones de los ríos Patía, Chicamocha, Cauca y además en el valle del río Magdalena ${ }^{1}$, mientras que en Cuba abunda en las sabanas arcillosas del sur de Pinar del Río².

En las hojas y en los tallos de Sapindus saponaria L. se han detectado los flavonoides, luteolín y rutín y la presencia de saponinas y taninos en el pericarpio del fruto. La semilla contiene los triterpenos y el esterol beta-sitosterol. En las partes aéreas se han detectado el stigmasterol, el ácido oleanólico, la luteolina, el orientin, el isoorientin, la luteolina-7-0-囚-glucurónido y la rutina ${ }^{3}$.

Sobre la base de lo antes planteado este trabajo tiene como objetivo: determinar los principales componentes de la planta Sapindus saponaria $L$ que crece en Cuba mediante el tamizaje fitoquímico de las diferentes partes de la planta.

\footnotetext{
Instituto de Farmacia y Alimentos, Universidad de la Habana, Cuba.

${ }^{2}$ Grupo de las Industrias de la Biotecnologia y Farmaceuticas de Cuba (BioCubafarma). La Habana. Cuba.
} 


\section{Materiales y métodos}

\section{Recolección y preparación del material vegetal}

El material vegetal se colectó en el área del Bosque Semicaducifolio del Jardín Botánico Nacional. Se recolectaron los frutos, la corteza del tallo y las hojas. Una parte de la muestra se depositó en el herbario del Jardín Botánico Nacional para la posterior herborización de la planta.

Las partes del material vegetal afectadas por insectos o con signos de daño se desecharon, las otras se lavaron con abundante agua corriente para eliminar los restos de tierra, seguido de tratamiento con hipoclorito de sodio al 0,5\% durante 5 a 10 minutos y enjuague posterior con abundante agua destilada. A continuación se secaron sobre papel de filtro a temperatura ambiente y se conservaron en una desecadora para su posterior procesamiento y análisis.

\section{Tamizaje fitoquímico y preparación de los extractos}

El material vegetal, hojas, pericarpio del fruto y corteza del fruto, fue procesado de forma independiente para la preparación de los extractos. Las hojas secas fueron molidas en molino de cuchillas, mientras que el pericarpio y tallos se maceraron hasta obtener una masa homogénea que se pesó, utilizando una balanza analítica (Sartorius AG, China). Para la extracción e identificación de los metabolitos secundarios en los extractos, se siguió la marcha experimental que se propone en el Manual de Prácticas de Laboratorio de Farmacognosia y Productos Naturales de la Universidad de la Habana ${ }^{4}$. La figura 1 resume en un diagrama dicha marcha, que, brevemente, consistió en una extracción secuencial de los componentes en el material vegetal utilizando inicialmente éter etílico, etanol y finalmente agua.

\section{Ensayos para la identificación cualitativa de metabolitos} secundarios

La identificación cualitativa de los metabolitos secundarios en los diferentes extractos (etéreos, alcohólicos y acuosos) de las partes de la planta estudiadas, se realizó siguiendo
Los procedimientos del Manual de Prácticas de Laboratorio de Farmacognosia y Productos Naturales de la Universidad de la Habana ${ }^{4}$ como se muestra continuación:

\section{Determinación de alcaloides. Ensayo de Dragendorff.}

Se tomó una alícuota del extracto y se añadió una gota de ácido clorhídrico concentrado. Se calentó suavemente y se dejó enfriar hasta que el pH se tornó en valores ácidos, posteriormente se añadieron 3 gotas del reactivo de Dragendorff. Los resultados del ensayo se consideraron positivos si se apreciaron la siguientes características: Opalescencia (+), turbidez definida $(++)$, precipitado $(+++)$.

\section{Determinación de azúcares reductores. Ensayo de Fehling.}

Se adicionó a una alícuota del extracto $2 \mathrm{~mL}$ de reactivo y se calentó en baño de agua de 5 a 10 minutos. El ensayo se consideró positivo $(+++)$ si la solución tomó coloración roja o apareció un precipitado rojo.

\section{Determinación de saponinas. Ensayo de la espuma}

Se tomó una alícuota del extracto y se diluyó en 5 veces su volumen en agua, agitándose fuertemente el tubo de ensayo, de 5 a 10 minutos. El ensayo se consideró positivo (+++) si apareció una espuma de $2 \mathrm{~mm}$ de espesor como mínimo y persistente por más de 2 minutos.

\section{Determinación de flavonoides. Ensayo de Shinoda.}

Se tomó una alícuota del extracto y se añadió $1 \mathrm{~mL}$ de ácido clorhídrico concentrado y una pequeña porción de cinta de magnesio metálico. Después de 5 minutos se añadió $1 \mathrm{~mL}$ de alcohol amílico. Se consideró un ensayo positivo (+++) cuando el alcohol amílico se coloreó de amarillo, naranja, carmelita o rojo intenso en todos los casos.

\section{Determinación de compuestos fenólicos y/o taninos. Ensayo de cloruro férrico.}

A una alícuota del extracto se añadió acetato de sodio para neutralizar y 3 gotas de una solución de tricloruro férri-

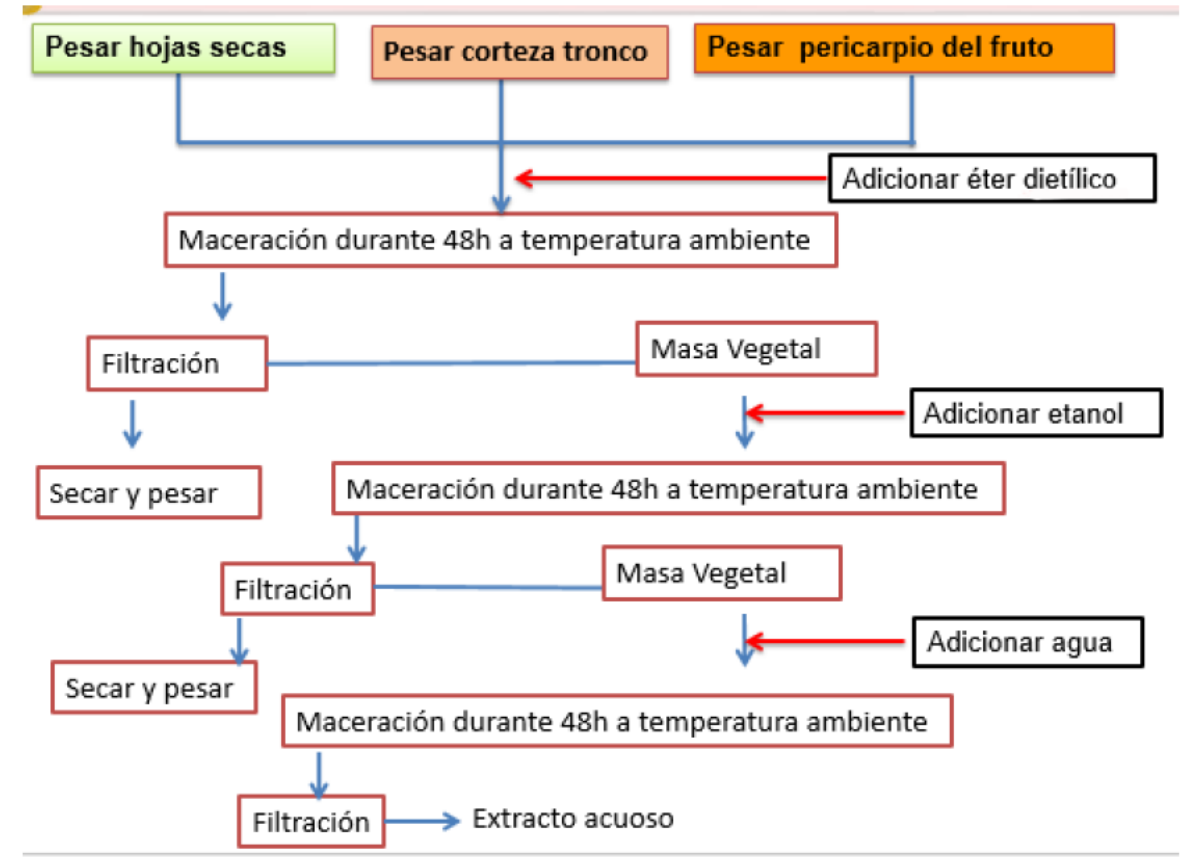

Figura 1. Diagrama que resume la marcha experimental del tamizaje fitoquímico realizado a las hojas de Sapindus saponaria L., que crece en Cuba 
co al 5\% en solución salina fisiológica. El ensayo se consideró positivo (+++) para compuestos fenólicos en general, al observarse un cambio de coloración a rojo-vino; para taninos del tipo pirocatecólicos una coloración verde intensa y para taninos del tipo pirogalotánicos una coloración azul.

\section{Determinación de secuencia C6-C3-C6 en flavonoides. Ensayo de antocianidina.}

Se calentaron $2 \mathrm{~mL}$ del extracto alcohólico por 10 min con $1 \mathrm{~mL}$ de $\mathrm{HCl}$ concentrado. Se dejó enfriar y se adicionó $1 \mathrm{~mL}$ de $\mathrm{H}_{2} \mathrm{O}$ y $2 \mathrm{~mL}$ de alcohol amílico. Se agitó y se dejaron separar las dos fases. La aparición de coloración roja o marrón en la fase amílica indica que el ensayo es positivo

\section{Determinación de estructuras del tipo polisacarídicas. Ensayo de mucílagos.}

Una alícuota del extracto se enfrió a una temperatura de $0^{\circ} \mathrm{C}$ a $5^{\circ} \mathrm{C}$ y se observó si la solución tomó una consistencia gelatinosa. El ensayo se consideró positivo $(+++)$ si la muestra tomó consistencia gelatinosa.

\section{Determinación de triterpenos y esteroides. Ensayo de Liebermann-Burchard.}

Se adicionó $1 \mathrm{~mL}$ de anhídrido acético y se mezcló bien. Por la pared del tubo de ensayos se dejaron caer 3 gotas de ácido sulfúrico concentrado sin agitar. El ensayo es positivo si el cambio de coloración es rápido; de rosado a azul muy rápido $(+++)$, a verde intenso visible rápido $(++)$, a verde oscuro-negro al final de la reacción (+).

Este método permite diferenciar las estructuras esteroidales de las triterpenoides, las primeras producen coloraciones azul o azul verdoso, mientras que en las segundas se observa rojo, rosado o púrpura.

\section{Determinación de aminoácidos libres o aminas. Ensayo de Ninhidrina.}

Se tomó una alícuota del extracto alcohólico se mezcló con $2 \mathrm{~mL}$ de solución de Ninhidrina al $2 \%$ en agua. La mezcla se calentó 5-10 minutos en baño de agua. Este ensayo se considera positivo cuando se desarrolla una coloración azul violácea.

\section{Determinación de Quinonas. Ensayo de Borntanger.}

Se evaporó el extracto alcohólico en baño de agua y el residuo se re-disolvió en $1 \mathrm{~mL}$ de cloroformo, luego se adicionó $1 \mathrm{~mL}$ de hidróxido de sodio al $5 \%$ en agua, posteriormente se agitó mezclando las fases, y se dejó en reposo hasta su separación. El ensayo se considera positivo si la fase acuosa alcalina (superior) se colorea de rosado (++) o rojo (+++).

\section{Cuantificación de Saponinas}

\section{Ensayo de hemólisis de eritrocitos}

La determinación cuantitativa de saponinas en los extractos se realizó modificando la técnica descrita en el protocolo "Red Blood Cell Test System" y adaptada por Tamargo y Sierra ${ }^{5}$.

\section{Obtención y preparación de la sangre}

La sangre fue colectada de carneros saludables procedentes del CENPALAB, se utilizó tubos plásticos de centrífuga (Corning, EUA) que contenían $5 \mathrm{~mL}$ de tampón citrato en los cuales se adicionaron $45 \mathrm{~mL}$ de sangre por cada uno, se homogeneizó suave, para obtener a última hora una concentración 1:10 citrato: sangre.

\section{Aislamiento de los eritrocitos y obtención de la suspensión}

Se centrifugaron $2 \mathrm{~mL}$ de sangre a $1500 \times$ g por $15 \mathrm{mi}$ nutos a temperatura ambiente, se aplicó una centrífuga para viales Eppendorf (Heraesus, Alemania). Se extrajo el sobrenadante de la superficie al cuidado y el precipitado se lavó 4 veces con Tampón Fosfato Salino isotónico, pH 7,4 (TFSi). La determinación del número de eritrocitos en suspensión se realizó mediante conteo en cámara de Neubauer, para lo cual se tomó un alícuota de la suspensión de eritrocitos, se realizaron diluciones seriadas hasta 1/100 000 en TFSi, se calculó el número total de células para $1 \mathrm{~mL}$ de la suspensión y finalmente el volumen necesario para obtener $8 \times 10^{9}$ células en suspensión.

\section{Preparación de las muestras}

Se disolvió cada una de las muestras liofilizadas de los extractos de tallo, semilla y fruto en el volumen de TFSi necesario para obtener disoluciones a concentraciones de $1 \mathrm{mg} / \mathrm{mL}$ en cada caso. Paralelo se preparó una disolución de saponina patrón (SIGMA, EUA) Quillaja saponaria Molina a una concentración de 0,5 mg/mL en TFSi.

\section{Procedimiento del ensayo}

En viales independientes Eppendorf de 1,5 mL se pipetearon los volúmenes siguientes de cada muestra de extracto y de la saponina patrón al respecto: 0, 10, 20, 30, 40, 50, 60, 70 y $80 \mu \mathrm{L}$. Detrás se completó cada vial con TFSi hasta alcanzar un volumen final de $975 \mu \mathrm{L}$. En otros dos viales independientes se añadieron $975 \mu \mathrm{L}$ de agua destilada y $975 \mu \mathrm{L}$ de TFSi, para obtener el $100 \%$ y el $0 \%$ de hemólisis, respectivamente. A continuación se añadió una alícuota de $25 \mu \mathrm{L}$ de la suspensión de eritrocitos que contenía un aproximado de $8 \times 109$ células/ $\mathrm{mL}$ a cada vial y se incubaron por 10 minutos a temperatura ambiente, se agitó suave, en una zaranda (Stuart Scientific, Inglaterra). De seguida, se centrifugaron a 1500 × g durante un minuto, $300 \mu \mathrm{L}$ del sobrenadante, se depositaron en una placa de 96 pozos de fondo plano (Costar) y en conclusión se determinó la absorbancia de las muestras y el patrón a 540 nm, en un lector de placas (Amersham, EUA).

\section{Resultados y discusión}

El tamizaje fitoquímico se basa fundamentalmente en la identificación de los metabolitos secundarios presentes en los extractos de productos naturales, a través de reacciones y análisis químicos bien descritos en la literatura. El tamizaje fitoquímico se le realiza consecutivamente a los extractos etéreo, alcohólico y acuoso del producto natural con el fin de identificar y comparar los metabolitos secundarios extraídos con cada disolvente de diferentes polaridades.

Los resultados de los tamizajes fitoquímico de los extractos etéreo, alcohólico y acuoso de las hojas de $S$. saponaria $L$ que crece en Cuba se muestran en la tablal.

En los tamizajes fitoquímicos realizados a las hojas con los 3 disolventes utilizados, se sugiere la presencia de abundantes compuestos grasos, alcaloides, azúcares reductores, saponinas, compuestos fenólicos, pues con el ensayo de cloruro férrico se apreció el desarrollo de una coloración verde intensa como está descrito para un resultado positivo para taninos pirocatecólicos, aminoácidos libres o aminas, flavonoides, antocianinas y principios amargos. No se detectan agrupamientos lactónicos, catequinas, glicósidos cardiotónicos, mucílagos, triterpenos/esteroides, quinonas, benzoquinonas ni resinas 


\begin{tabular}{|l|l|c|c|c|}
\hline \multirow{2}{*}{ Hojas de Sapindus saponaria L. } & $\begin{array}{c}\text { Extracto } \\
\text { etéreo }\end{array}$ & $\begin{array}{c}\text { Extracto } \\
\text { alcohólico }\end{array}$ & $\begin{array}{c}\text { Extracto } \\
\text { acuoso }\end{array}$ \\
\hline Ensayos & Metabolitos & ++ & $\mathrm{X}$ & $\mathrm{X}$ \\
\hline Sudán & Compuestos grasos & - & +++ & +++ \\
\hline Dragendorff & Alcaloides & - & - & $\mathrm{X}$ \\
\hline Baljet & Agrupamiento lactónico & - & - & $\mathrm{X}$ \\
\hline Libermanm. B & Triterpenos/esteroides & $\mathrm{X}$ & - & $\mathrm{X}$ \\
\hline Catequinas & Catequinas & $\mathrm{X}$ & - & - \\
\hline Resinas & Resinas & $\mathrm{X}$ & +++ & +++ \\
\hline Fehling & Azúcares reductores & $\mathrm{X}$ & +++ & +++ \\
\hline Espuma & Saponinas & $\mathrm{X}$ & +++ & +++ \\
\hline Cloruro Fe & Compuestos fenólicos & $\mathrm{X}$ & - & +++ \\
\hline Ninhidrina & Aminoácidos libres ó & & & \\
\hline aminas & Quinonas benzoquino & $\mathrm{X}$ & - & $\mathrm{X}$ \\
\hline Borntrager & Flavonoides & $\mathrm{X}$ & +++ & +++ \\
\hline Shinoda & Glicósidos cardiotónicos & $\mathrm{X}$ & - & $\mathrm{X}$ \\
\hline Kedde & Antocianinas & $\mathrm{X}$ & +++ & $\mathrm{X}$ \\
\hline Antocianidina & Mucílagos & $\mathrm{X}$ & $\mathrm{X}$ & - \\
\hline Mucílagos & Principios amargos & $\mathrm{X}$ & $\mathrm{X}$ & +++ \\
\hline $\begin{array}{l}\text { Principios } \\
\text { amargos }\end{array}$ & & & & \\
\hline
\end{tabular}

Tabla 1. Resultado del tamizaje fitoquímico de los extractos etéreo, alcohólico y acuoso de las hojas de Sapindus saponaria L., que crece en Cuba.

Tabla 2. Resultados del tamizaje fitoquímico de los extractos etéreo, alcohólico y acuoso de la corteza del tallo de Sapindus saponaria $L_{\text {., }}$ que crece en Cuba.

Leyenda: + Presencia escasa, ++ Presencia relativamente abundante, +++ Presencia abundante, No detectado, X No realizado.

\begin{tabular}{l|l|c|c|c}
\hline \multirow{2}{*}{$\begin{array}{l}\text { Corteza del tallo de Sapindus saponaria L. } \\
\text { Ensayos }\end{array}$} & Metabolitos & \multicolumn{3}{c}{$\begin{array}{c}\text { Resultados } \\
\text { etéreo } \\
\text { alcohólico }\end{array}$} \\
\hline Sudan & Compuestos grasos & ++ & X & X \\
\hline Dragendorff & Alcaloides & - & +++ & + \\
\hline Baljet & Agrupamiento lactónico & +++ & - & X \\
\hline Libermanm. B & Triterpenos/esteroides & - & - & X \\
\hline Catequinas & Catequinas & $\mathrm{X}$ & - & $\mathrm{X}$ \\
\hline Resinas & Resinas & $\mathrm{X}$ & +++ & - \\
\hline Fehling & Azúcares reductores & $\mathrm{X}$ & +++ & +++ \\
\hline Espuma & Saponinas & $\mathrm{X}$ & +++ & + \\
\hline Cloruro Fe & Compuestos fenólicos & $\mathrm{X}$ & +++ & +++ \\
\hline Ninhidrina & Aminoácidos libres ó & $\mathrm{X}$ & +++ & $\mathrm{X}$ \\
\hline Borntrager & Quinonas benzoquino & $\mathrm{X}$ & - & $\mathrm{X}$ \\
\hline Shinoda & Flavonoides & $\mathrm{X}$ & +++ & +++ \\
\hline Kedde & Glicósidos cardiotónicos & $\mathrm{X}$ & - & $\mathrm{X}$ \\
\hline Antocianidina & Antocianinas & $\mathrm{X}$ & +++ & $\mathrm{X}$ \\
\hline Mucílagos & Mucílagos & $\mathrm{X}$ & $\mathrm{X}$ & - \\
\hline $\begin{array}{l}\text { Principios } \\
\text { amargos }\end{array}$ & Principios amargos & $\mathrm{X}$ & $\mathrm{X}$ & + \\
\hline & & & & \\
\hline
\end{tabular}

Leyenda: + Presencia escasa, ++ Presencia relativamente abundante, +++ Presencia abundan No detectado, X No realizado.

Los resultados de los tamizajes fitoquímicos de los extractos de la corteza del tallo antes de liofilizar se muestran en la tabla 2.

En los tamizajes fitoquímicos realizados a la corteza del tallo con los 3 disolventes utilizados, se sugiere la presencia de abundantes compuestos grasos, alcaloides, azúcares reductores, agrupamientos lactónicos, aminoácidos libres o aminas, saponinas, compuestos fenólicos, flavonoides, antocianinas y resinas. Se detectaron escasos principios amargos. No se detectan catequinas, triterpenos/esteroides, quinonas benzoquinonas, mucílagos, principios amargos ni glicósidos cardiotónicos.

Los resultados de los tamizajes fitoquímicos de los extrac- tos etéreo, alcohólico y acuoso del pericarpio del fruto se muestran en la tabla 3.

En los tamizajes fitoquímicos realizados a los extractos de Sapindus saponaria L. del pericarpio del fruto con los 3 disolventes utilizados, se sugiere la presencia de azúcares reductores, aminoácidos libres o aminas, saponinas, taninos, pirocatecolicos, flavonoides y triterpenos/esteroides, este último solo para el extracto con éter dietílico.

Por otro lado, no se detectan compuestos grasos, alcaloides, agrupamientos lactónicos y catequinas, ni glicósidos cardiotónicos. 


\begin{tabular}{|l|l|c|c|c|}
\hline \multirow{2}{*}{$\begin{array}{l}\text { Pericarpio del fruto de Sapindus saponaria L. } \\
\text { Ensayos }\end{array}$} & Metabolitos & $\begin{array}{c}\text { Extracto } \\
\text { etéreo }\end{array}$ & $\begin{array}{c}\text { Extracto } \\
\text { alcohólico }\end{array}$ & $\begin{array}{c}\text { Extracto } \\
\text { acuoso }\end{array}$ \\
\hline Sudan & Compuestos grasos & + & $\mathrm{X}$ & - \\
\hline Dragendorff & Alcaloides & - & - & - \\
\hline Baljet & Agrupamiento lactónico & - & - & - \\
\hline Libermanm. B & Triterpenos/esteroides & +++ & + & - \\
\hline Catequinas & Catequinas & $\mathrm{X}$ & - & - \\
\hline Resinas & Resinas & $\mathrm{X}$ & +++ & +++ \\
\hline Fehling & Azúcares reductores & $\mathrm{X}$ & +++ & +++ \\
\hline Espuma & Saponinas & $\mathrm{X}$ & +++ & +++ \\
\hline Cloruro Fe & Compuestos fenólicos & $\mathrm{X}$ & +++ & +++ \\
\hline Ninhidrina & Aminoácidos libres ó aminas & $\mathrm{X}$ & +++ & ++ \\
\hline Borntrager & Quinonas benzoquino & $\mathrm{X}$ & - & - \\
\hline Shinoda & Flavonoides & $\mathrm{X}$ & - & - \\
\hline Kedde & Glicósidos cardiotónicos & $\mathrm{X}$ & - & - \\
\hline Antocianidina & Antocianinas & $\mathrm{X}$ & - & - \\
\hline
\end{tabular}

Leyenda: + Presencia escasa, ++ Presencia relativamente abundante, +++ Presencia abundante, No detectado, X No realizado.

Tabla 3. Resultados del tamizaje fitoquímico de los extractos etéreo, alcohólico y acuoso del pericarpio del fruto de Sapindus saponaria L. que crece en Cuba.

\section{Ensayo de Hemolisis}

El ensayo de hemólisis, también conocido como ensayo de fragilidad osmótica, analiza el efecto de la concentración de saponinas en la actividad hemolítica. Las saponinas pueden producir hemólisis a grandes diluciones, por lo que resultan tóxicas para el ser humano administradas directamente al torrente sanguíneo ${ }^{6}$.

La figura 2 muestra las gráficas de actividad hemolítica en \% versus el logaritmo de concentración de cada uno de los extractos liofilizados de pericarpio del fruto, hojas y corteza del tallo de Jaboncillo.

La actividad hemolítica sobre los eritrocitos de carnero, de los extractos acuosos de Jaboncillo liofilizados, preparados a una concentración de $1 \mathrm{mg} / \mathrm{mL}$ en TFSi, es dependiente de la concentración del extracto, por tanto, a mayor concentración, mayor actividad hemolítica.

El extracto del pericarpio del fruto fue el que mostró mayor actividad hemolítica media (CH50), siendo la concentración de 101,4 $\mu \mathrm{g} / \mathrm{mL}$ la que provoca el 50\% de hemólisis de los eritrocitos de carnero. Mientras que, los extractos de las hojas y de la corteza del tallo no provocan la hemólisis de los eritrocitos, al menos en las concentraciones ensayadas.

Estos resultados se corresponden con los obtenidos en el tamizaje fitoquímico donde se obtuvo que, el extracto del pe- ricarpio del fruto posee mayor cantidad de saponinas (referido a la prueba de la espuma) mientras que, en los extractos de hojas y de corteza del tallo, se detectaron las saponinas en menor cantidad.

Los resultados obtenidos coinciden con los de Díaz, 2015², cuando analizó la actividad hemolítica de extractos de semillas de Chenopodium quinoa W. y demostró que a mayor concentración de saponinas es mayor la actividad hemolítica media.

Por otro lado los resultados de la evaluación de la CH50 de los extractos de la $S$. saponaria $L$ que crece en Cuba, difieren con los obtenidos por Mena ${ }^{8}$, que al analizar extractos acuosos de pericarpio del fruto, semillas y corteza del tallo de la planta Sapindus saponaria L., que crece en Ecuador en la provincia de Manabí, encontró que los extracto del pericarpio del fruto, semilla y corteza del tallo causaron el 50\% de hemólisis de los eritrocitos a concentraciones mucho menores $(1,72,59,5$ y $150,9 \mu \mathrm{g} / \mathrm{mL}$ respectivamente) que los encontrados, para los extractos acuosos del pericarpio del fruto y de la corteza del tallo de la planta que crece en Cuba, preparados a la misma concentración $(1 \mathrm{mg} / \mathrm{mL})$. Por lo que, los extractos de Jaboncillo que crece en Ecuador resultaron tener mayor cantidad de saponinas y ser en correspondencia con ello, más hemo tóxicos que los de la misma planta recolectada en nuestro
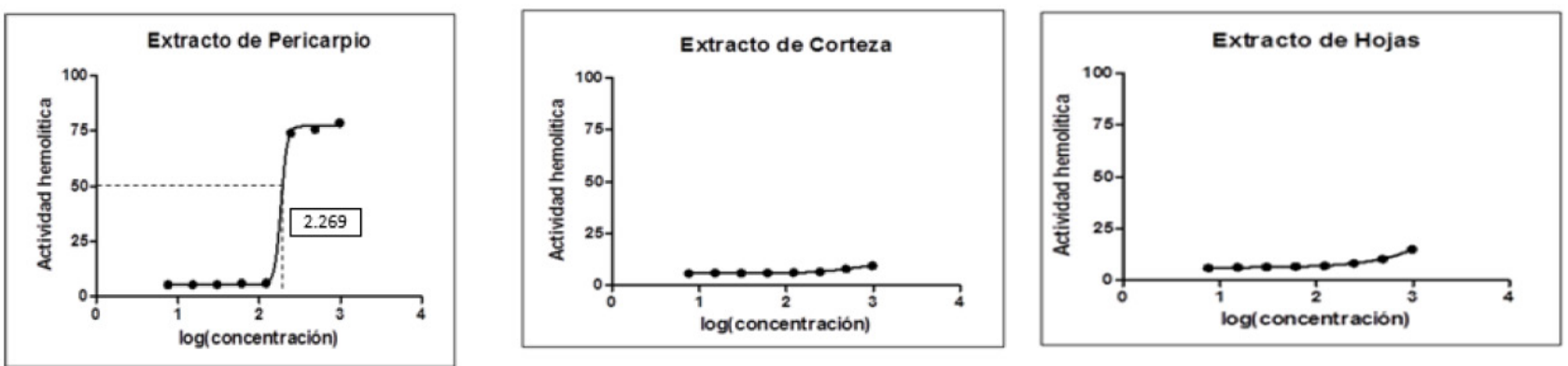

Figura 2. Actividad hemolítica media de extractos acuosos de Sapindus saponaria L., que crece en Cuba. 
país. La diferencia entre los resultados encontrados pudiera deberse a que las condiciones climáticas, altitud, composición de nutrientes del suelo, luz solar y otros factores que inciden en la producción de los metabolitos secundarios de las plantas, no son las mismas por tanto en condiciones diferentes de crecimiento, las plantas no producen los mismos metabolitos secundarios, ni en las mismas proporciones ${ }^{9}$.

Si bien las propiedades surfactantes de las saponinas favorecen el uso popular de estas especies, también le confieren una actividad biológica muy peculiar que es la capacidad de destruir las membranas de los eritrocitos y causar la hemólisis de los mismos. Tal efecto fue observado para los extractos acuosos del pericarpio del fruto y en menor medida en el de las hojas y corteza del tallo de Sapindus saponaria L. evaluados en este trabajo y mostrado los resultados en la figura 2. La actividad hemolítica ha sido observada por muchos investigadores no solo para los extractos de Sapindus saponaria L. sino también para extractos de otras plantas que igual a esta son ricas en saponinas ya sean esteroidales o triterpénicas ${ }^{5}$.

\section{Conclusiones}

Como consideraciones finales de este trabajo debemos decir que el tamizaje fitoquímico mostró la presencia de metabolitos secundarios en hojas, corteza del tallo y pericarpio del fruto de la planta siendo, los de mayor abundancia saponinas, flavonoides, azúcares reductores y taninos, lo cual pudiera estar relacionado con las propiedades medicinales que se le atribuyen a la planta ${ }^{10,11}$. Así mismo, las saponinas y flavonoides son los metabolitos secundarios de mayor representatividad en todos los extractos acuosos de las partes de la planta, siendo más abundante las saponinas en el pericarpio del fruto y los flavonoides en las hojas.

Teniendo en cuenta la composición de los extractos se plantean promisorias las evaluaciones farmacológicas de la planta Sapindus saponaria $L$ que crece en Cuba ante diversas patologías.

\section{Referencias bibliográficas}

1. Cogollo KA. Bondades del fruto del jaboncillo (Sapindus saponaria) como un detergente biodegradable [Tesis de pregrado]. Instituto Alexander Von Humboldt: Departamento de Ciencias Básicas. Barranquilla, Colombia; 2008.

2. Roig JT. Plantas medicinales, aromáticas o venenosas de Cuba, Segunda edición. Editorial Científico Técnica. La Habana; 2012.

3. Tomás G, Huamán J, Aguirre R, Barreram T. Extracción y clasificación de la saponina del Sapindus saponaria. Revista Peruana de Química e Ingeniería Química, 2014; 13 (2): 36-39.

4. Miranda, M C. Manual de prácticas de laboratorio, farmacognosia y productos naturales. Universidad de la Habana, 2000

5. Tamargo B, Sierra GV. Sapindus saponaria L. Actividad antiproliferativa: Extractos acuosos de la planta Sapindus saponaria L. Metabolitos secundarios y actividad antiproliferativa. Editorial Académica Española; 2016.

6. Lorent JH, Quetin J, Mingeot MP. The amphiphilic nature of saponins and their effects on artificial and biological membranes and potential consequences for red blood and cancer cells. Org Biomol Chem. 2014; 12(44): 8803-22.

7. Díaz L. Saponinas y otros metabolitos secundarios en semillas de Chenopodium quinoa [Tesis de pregrado]. Instituto de Farmacia y Alimentos. Universidad de la Habana,Cuba; 2015.

8. Mena L. Actividad hemolítica y antiproliferativa de los extractos acuosos de la planta Sapindus saponaria L [Tesis de pregrado]. Instituto de Farmacia Alimentos, Universidad de La Habana; 2013.

9. Wang L. Exploration of the correlation between the structure, hemolytic activity, and cytotoxicity of steroid saponins. Bioorganic \& Medicinal Chemistry, 2008; 15: 2528-2532

10. Abreu O. Potencial medicinal del género Sapindus L. (Sapindaceae) y de la especie Sapindus saponaria L. Rev. Cub. Plant. Med, 2005; 10: 3-4.

11. Ahumada A. Saponinas de quinua (Chenopodium quinoa Wild.): un subproducto con alto potencial biológico, Rev. Colomb. Cienc. Quím. Farm. 2016; 45(3): 438-469.

Received: 25 junio 2020

Accepted: 20 julio 2020 